\title{
The Relationship between Autonomic Regulation of Cardiovascular Function and Body Composition
}

\author{
Tomislav Smoljo ${ }^{1}$, Ivan Stanić', Sara Sila ${ }^{2}$, Uroš Kovačić3 ${ }^{3}$ Luka Crnošija ${ }^{4}$, Anamari Junakovićc, Ivan Adamec ${ }^{4}$, Iva Hojsak ${ }^{1,2,5}$, \\ Magdalena Krbot Skoricici, ${ }^{4}$ Mario Habek ${ }^{1,4, *}$ \\ 'Department of Neurology, School of Medicine, University of Zagreb, Zagreb; ${ }^{2}$ Children's Hospital Zagreb, Zagreb, Croatia; ${ }^{3}$ Institute of Pathophysiology, Faculty of \\ Medicine, University of Ljubljana, Ljubljana, Slovenia; ${ }^{4}$ Department of Neurology, Referral Center for Autonomic Nervous System Disorders, University Hospital \\ Center Zagreb, Zagreb; ${ }^{5}$ Department of Pediatrics, Josip Juraj Strossmayer University of Osijek, Osijek; ${ }^{6}$ Faculty of Electrical Engineering and Computing, University of \\ Zagreb, Zagreb, Croatia
}

Background: We investigated whether the results of autonomic function tests correlate with body composition and shape in healthy young people.

Methods: We conducted cardiovascular reflex tests (heart rate [HR] and blood pressure [BP] responses to the Valsalva maneuver and HR response to deep breathing) and the tilt table test with 32 subjects (19 males; mean age, $22.1 \pm 1.9$ years). Participants also completed an anthropometric measurement sequence (weight; height; upper arm, hips, and waist circumference; triceps and subscapular skinfold), bioelectric impedance testing, and hand grip strength measurements.

Results: Markers of obesity, other anthropometric measures, functional measures, and the basal metabolic rate (BMR) were significantly positively correlated with systolic $\mathrm{BP}(\mathrm{SBP})$ and diastolic $\mathrm{BP}(\mathrm{DBP})$ in both the supine and tilted positions. There was a positive correlation between the difference in $H R(\triangle H R)$ between the tilt and supine body positions and markers of obesity, the functional marker of dominant handgrip strength, and BMR. Participants with a body mass index (BMI) $<25 \mathrm{~kg} / \mathrm{m}^{2}$ had significantly lower median values of $\triangle H R$, DBP in the tilttest, SBP at rest, and SBP in the tilt-test than participants who had a BMl $\geq 25 \mathrm{~kg} / \mathrm{m}^{2}$ (10.55 vs. $21.95 \mathrm{bpm}$, $P=0.003 ; 77.55$ vs. $90.05 \mathrm{mmHg}, P=0.045 ; 113.45$ vs. $140.55 \mathrm{mmHg}, P=0.013 ; 117.00$ vs. $135.25 \mathrm{mmHg}$, $P=0.006$, respectively). Body fat percentage was identified as an independent positive predictor ( $\beta=0.993 ; 95 \%$ confidence interval $[\mathrm{Cl}], 0.070$ to $1.916 ; P=0.036$ ) and body water percentage was an independent negative predictor of tilted SBP $(\beta=-1.370 ; 95 \% \mathrm{Cl},-2.634$ to $0.106 ; P=0.035)$.

Conclusion: High sympathetic activity, as evaluated by cardiovascular regulation, correlates with a high share of adipose tissue in young healthy persons.

Key words: Autonomic nervous system, Fat tissue, Body composition, Heart rate, Blood pressure, Heart rate variability, Cardiovascular reflexes
Received May 5, 2020

Reviewed June 15, 2020

Accepted August 10, 2020

*Corresponding author Mario Habek

(iD) https://orcid.org/0000-0002-3360-1748

Department of Neurology, University Hospital Center Zagreb, Kišpatićeva 12, HR-10000 Zagreb, Croatia Tel: +385-12388033

Fax: +385-12388033 E-mail:mhabek@mef.hr

\section{INTRODUCTION}

The autonomic nervous system (ANS) regulates many functions, including circulation and metabolism. The ANS exerts direct control of adiposity at the cellular and molecular levels. Both the sympathetic nervous system (SNS) and the parasympathetic nervous system (PNS) provide complex homeostatic control to specifically coordinate the function of adipose tissue; the SNS stimulates catabolism, and the PNS stimulates anabolism. ${ }^{1}$ Because the ANS regulates fat distribution and formation, it can influence body shape and composition. ${ }^{2}$ This has been proposed as a possible explanation for the correlation between abdominal obesity (central obesity)

Copyright (C) 2020 Korean Society for the Study of Obesity

(a) This is an Open Access article distributed under the terms of the Creative Commons Attribution Non-Commercial License (https://creativecommons.org/licenses/by-nc/4.o/) which permits unrestricted non-commercial use, distribution, and reproduction in any medium, provided the original work is properly cited. 
and hypertension, dyslipidemia, and hyperglycemia, which could all appear as a result of prolonged high SNS activity. ${ }^{3}$ It has been shown that obesity and central body adiposity correlate with alterations in the ANS, and therefore, people at greater risk for those conditions could be tested to see whether their ANS is distorted. ${ }^{4-6}$

The ANS and body composition, which provides a specific image of metabolism, are connected and affect the same diseases, such as hypertension, dyslipidemia, hyperglycemia and many other acquired chronic diseases that can be treated only symptomatically. Interventions that affect both metabolism and body composition, such as exercise, act synergistically with pharmacological therapy using beta receptor blockers. ${ }^{8,9}$ Research on this topic has included older people ${ }^{10}$ and children ${ }^{11}$ and focused on conditions such as obesity, ${ }^{12,13}$ malnutrition, ${ }^{14}$ and pregnancy. ${ }^{15}$

In healthy individuals, an increase in the body mass index (BMI) correlates with increased sympathetic activity and lowered parasympathetic activity. ${ }^{16}$ The identification of a link between ANS activity and BMI could provide valuable insights into the physiological regulation of body weight (BW) and important clues for the development of effective anti-obesity strategies. ${ }^{17}$ Because most previously published studies did not make a thorough nutritional assessment and used only specific tests of the ANS (usually heart rate variability $[\mathrm{HRV}])$, our aim in this study was to determine whether standardized autonomic function tests correlate with body composition and shape, as evaluated with a thorough nutritional assessment, in healthy young people.

The primary objective of this study was to investigate the correlation between the results from ANS tests and body composition and constitution. The secondary objectives were to investigate differences in heart rate (HR), systolic blood pressure (SBP), and diastolic BP (DBP) using a BMI cutoff of $25 \mathrm{~kg} / \mathrm{m}^{2}$ to indicate overweight individuals.

\section{METHODS}

\section{Participants}

Thirty-two healthy subjects (19 males and 13 females; mean age, $22.1 \pm 1.9$ years) took part in this study. The Croatian version of the Composite Autonomic Symptom Score 31 (COMPASS 31) questionnaire was used to evaluate autonomic symptoms. ${ }^{18}$ The
Godin Leisure-Time Exercise Questionnaire, a self-explanatory, four-item query, was used to evaluate their leisure-time exercise habits. The test group had no illnesses or disabilities, and they were all enlisted as healthy subjects. All participants were acquainted with the study protocol and provided informed consent. This study was approved by the Ethical Committee of University Hospital Center Zagreb (02/21 AG, January 30, 2018).

\section{ANS testing}

The ANS testing protocol was performed in the Referral Center for ANS disorders at University Hospital Center Zagreb. Subject preparation included dressing in comfortable clothes and then lying down on a tilt table (table that can be inclined). Electrocardiogram electrodes were attached, and sphygmomanometer cuffs were put around the right upper arm and the fingers of the left hand to measure BP at every heartbeat. Prior to the testing, safety belts were tightened to prevent injuries if syncope occurred during the tilt test. The test protocol consisted of the following: (1) 10-minute rest on the tilt table (supine phase), (2) three consecutive Valsalva maneuvers with a 3-minute pause after each maneuver, (3) a deep breathing test followed by a 3-minute pause, (4) 10-minute tilt table test, and (5) 5-minute rest period. Throughout the testing, BP and HR were continuously measured beat to beat using a task force monitor (TFM; Medizintechnik AG, Graz, Austria).

For the Valsalva maneuver, the test subject blew into a tube connected to the sphygmomanometer to maintain the pressure on the sphygmomanometer at $40 \mathrm{mmHg}$ for 15 seconds. A little hole was created on the tube to allow the air to leak and prevent the glottis from closing during the test. The test was repeated three times to get a repeatable response. ${ }^{19}$ The deep breathing test was performed in the supine position because vagal tone is the highest in that position. That test consists of six respiratory cycles lasting 1 minute. Each respiratory cycle consists of inhaling deeply and uniformly through nose for 5 seconds and then exhaling uniformly for $5 \mathrm{sec}$ onds through mouth. ${ }^{19}$

The tilt table test stimulates the ANS by orthostatic provocation. The test begins with the test subject lying in the supine position for 10 minutes. In controlled conditions the tilt table is inclined to $70^{\circ}$ so that the subject's head is up, and that position is maintained for 10 minutes, after which the table is lowered to the starting position. 
$\mathrm{BP}$ and heart frequency were monitored during the test. ${ }^{19}$ Difference in $\mathrm{HR}(\triangle \mathrm{HR})$ between the tilt and supine body positions, difference in SBP between the tilt and supine body positions $(\triangle \mathrm{SBP})$, and difference in DBP between the tilt and supine body positions $(\triangle \mathrm{DBP})$ denote the differences in the average values of those measurements in the tilt and supine phases of the tilt-table test $(\Delta=$ tilt-supine).

In this study, we used the Valsalva ratio (VR) and respiratory sinus arrhythmia (RSA) to evaluate impaired cardiovagal function. VR represents the ratio between the highest heart frequency during phase II and the lowest heart frequency during phase IV of the Valsalva maneuver. RSA is defined as a difference between the end inspiration heart frequency and the end expiration heart frequency. We calculated RSA for each of the six respiratory cycles of the deep breathing test and used the mean value in our analyses. ${ }^{20}$

HRV spectral analyses were performed using Kubios HRV 2.2 software (Department of Applied Physics, University of Eastern Finland, Kuopio, Finland). The autoregressive spectral analysis method was used to analyze the frequency domain parameters. Data used for the HRV analyses were recorded by TFM with a sampling rate of $1,000 \mathrm{~Hz}$. After the recordings were complete, the data were inspected and edited to correct for any missing data. The quality of the data was ensured by using the medium artefact correction option and smoothness priors-based detrending approach $($ Lambda $=500)$. HRV was analyzed in three 5-minute windows from the beat to beat data recorded during the test: the first $5 \mathrm{~min}$ utes, the 2 nd -7 th minute, and the last 5 minutes. During the first 10-minute rest period prior to the tilt-table test an HRV analysis was conducted on the most stable 5-minute interval for each test subject ("s" variable). The high frequency (HF) power (0.15-0.4 $\mathrm{Hz}$ ) of the RR intervals is expressed in absolute units and predominantly dependent on cardiovagal tonus, so we used it as an index of parasympathetic heart activity. The ratio of the low and high frequencies $(\mathrm{LF} / \mathrm{HF})$ was used as an expression of sympathetic-vagal balance. The standard deviation of the mean of qualified NN-interval (SDNN) and root mean square of the successive differences ( $r$ MSSD) time-domain parameters were used as total HRV markers, although at least r-MSSD is predominantly influenced by cardiovagal modulation. Another time domain parameter, proportion of NN50 divided by the total number of NN (R-R) intervals (pNN50), was used as index of parasympathetic activity. ${ }^{21}$

\section{Body composition determination}

Anthropometric measurements were assessed at the Referral Center for Pediatric Gastroenterology and Nutrition, Children's Hospital Zagreb. Each participant was subjected to a series of anthropometric measurements. BW, body height $(\mathrm{BH})$, middle upper arm circumference (MUAC), hip circumference (HC), waist circumference (WC), a body shape index (ABSI), triceps skinfold thickness (TST), subscapular skinfold thickness (SST), and handgrip strength (HS) were estimated by the same trained person. $\mathrm{BMI}$, waist to height ratio ( $\mathrm{W} / \mathrm{H}$ ratio), and waist to hip ratio (W/ Hip ratio) were calculated from those parameters. TST and SST were estimated using a Holtain skinfold caliper (Holtain Tanner/ Whitehouse Skinfold Caliper; Holtain, Crosswell, UK). A Jamar hydraulic handgrip dynamometer (J. A. Preston Corp., New York, NY, USA) was used to estimate both dominant (dHGS) and nondominant (nHGS) HS. Body composition (body fat percentage [BF\%], lean body mass percentage [LBM\%], body water percentage $[\mathrm{BW} \%$, and basal metabolic rate $[\mathrm{BMR}]$ ) was determined by bioelectrical impedance (Maltron BF906; Maltron International, Rayleigh, Essex, UK).

BMI was calculated as BW in kilograms divided by $\mathrm{BH}$ in meters squared $\left(\mathrm{kg} / \mathrm{m}^{2}\right)$. It was used to classify participants according to their nutritional status: underweight $\left(<18.5 \mathrm{~kg} / \mathrm{m}^{2}\right)$, normal weight $\left(\geq 18.5\right.$ and $\left.<25.0 \mathrm{~kg} / \mathrm{m}^{2}\right)$, overweight $\left(\geq 25 \mathrm{~kg} / \mathrm{m}^{2}\right)$, or obese $\left(\geq 30 \mathrm{~kg} / \mathrm{m}^{2}\right){ }^{22}$

\section{Statistical analysis}

We used IBM SPSS version 25 (IBM Corp., Armonk, NY, USA) for all statistical analyses. We used the Kolmogorov-Smirnov test to determine whether the data were normally distributed. In the numeric data analysis considering distribution, parametric ( $\mathrm{t}$-test for dependent samples) and non-parametric (Mann-Whitney test) statistical methods were used. Correlations between certain variables were examined using Pearson's or Spearman's coefficients. Univariable and multivariable linear regression analyses were used to determine which variables were statistically significant predictors for the tilted SBP. The level of statistical significance was set at $P<0.05$. 


\section{RESULTS}

\section{Descriptive analysis}

Descriptive results for all 32 subjects are presented in Supplementary Tables 1-5. Supplementary Table 1 presents the anthropometric measures; Supplementary Table 2 data are from COMPASS 31; Supplementary Table 3 contains the results of the ANS testing; Supplementary Table 4 presents the results of the HRV analysis; and Supplementary Table 5 contains a descriptive presentation of the Godin questionnaire results. By nutritional status, three participants were underweight, 21 had normal weight, six were overweight, and two were obese.

\section{Association of autonomic and anthropometric parameters}

Separate analyses were performed for the supine phase of ANS testing (Table 1), the tilt phase of ANS testing (Table 2), and differences in HR, SBP, and DBP between the tilt and supine phases of ANS testing overall (Table 3), by BMI (Table 4), and by the VR. Only statistically significant results are presented.

Table 1. Numerical results from analyzing the correlation between the anthropometric parameters and autonomic parameters during the supine phase of the tilttest

\begin{tabular}{lcr}
\hline Variable & Correlation coefficient & $P$ \\
\hline SBP & & \\
BMI & $r_{p}=0.696$ & $<0.001$ \\
BF\% & $r_{s}=0.526$ & 0.002 \\
SST & $r_{s}=0.476$ & 0.006 \\
W/H ratio & $r_{p}=0.464$ & 0.008 \\
W/Hip ratio & $r_{p}=0.389$ & 0.028 \\
BH & $r_{p}=0.518$ & 0.002 \\
BW & $r_{p}=0.706$ & 0.001 \\
WC & $r_{p}=0.654$ & $<0.001$ \\
HC & $r_{p}=0.709$ & $<0.001$ \\
MUAC & $r_{p}=0.627$ & $<0.001$ \\
nHGS & $r_{p}=0.357$ & 0.048 \\
LBM $\%$ & $r_{p}=-0.477$ & 0.006 \\
BW\% & $r_{p}=-0.535$ & 0.002 \\
\hline
\end{tabular}

Only statistically significant results are presented.

$r_{p}$, Pearson linear; $r_{s}$, Spearman rank; SBP, systolic blood pressure; BMl, body mass index; BF\%, body fat percentage; SST, subscapular skinfold thickness; W/H ratio, waist to height ratio; W/Hip ratio, waist to hip ratio; $\mathrm{BH}$, body height; $\mathrm{BW}$, body weight; $\mathrm{WC}$, waist circumference; $\mathrm{HC}$, hip circumference; MUAC, middle upper arm circumference; nHGS, nondominant handgrip strength; LBM\%, lean body mass percentage; BW\%, body water percentage.

\section{Markers of obesity correlate positively with supine SBP}

Markers of obesity (BMI, BF\%, SST, $\mathrm{W} / \mathrm{H}$ ratio, $\mathrm{W} /$ Hip ratio) were significantly and positively correlated with SBP. Other anthropometric measures (BH, BW, WC, HC, MUAC) and nHGS were also positively correlated with SBP. Respondents with higher LBM\% and BW\% had significantly lower SBP. These numerical results are presented in Table 1 . We found no significant correlations for supine DBP.

Table 2. Numerical results from analyzing the correlation between the anthropometric parameters and autonomic parameters during the tilt-up phase of the tilttest

\begin{tabular}{|c|c|c|}
\hline Variable & Correlation coefficient & $P$ \\
\hline \multicolumn{3}{|l|}{ SBP } \\
\hline $\mathrm{BMl}$ & $r_{p}=0.693$ & $<0.001$ \\
\hline $\mathrm{BF} \%$ & $r_{p}=0.508$ & 0.003 \\
\hline SST & $r_{s}=0.482$ & 0.005 \\
\hline W/Hip ratio & $r_{p}=0.442$ & 0.011 \\
\hline $\mathrm{BH}$ & $r_{p}=0.445$ & 0.011 \\
\hline BW & $r_{p}=0.688$ & $<0.001$ \\
\hline WC & $r_{p}=0.592$ & $<0.001$ \\
\hline $\mathrm{HC}$ & $r_{p}=0.554$ & 0.001 \\
\hline MUAC & $r_{p}=0.672$ & $<0.001$ \\
\hline $\mathrm{dHGS}$ & $r_{p}=0.497$ & 0.004 \\
\hline nHGS & $r_{p}=0.577$ & $<0.001$ \\
\hline BMR & $r_{p}=0.496$ & 0.004 \\
\hline LBM\% & $r_{p}=-0.475$ & 0.006 \\
\hline BW\% & $r_{p}=-0.512$ & 0.003 \\
\hline \multicolumn{3}{|l|}{ DBP } \\
\hline BMl & $r_{p}=0.636$ & $<0.001$ \\
\hline $\mathrm{BF} \%$ & $r_{p}=0.576$ & $<0.001$ \\
\hline SST & $r_{s}=0.409$ & 0.020 \\
\hline W/Hip ratio & $r_{p}=0.422$ & 0.016 \\
\hline $\mathrm{BH}$ & $r_{p}=0.363$ & 0.041 \\
\hline BW & $r_{p}=0.605$ & $<0.001$ \\
\hline WC & $r_{p}=0.559$ & $<0.001$ \\
\hline $\mathrm{HC}$ & $r_{p}=0.515$ & 0.003 \\
\hline MUAC & $r_{p}=0.535$ & 0.002 \\
\hline dHGS & $r_{p}=0.361$ & 0.046 \\
\hline nHGS & $r_{p}=0.390$ & 0.030 \\
\hline BMR & $r_{p}=0.390$ & 0.027 \\
\hline BW\% & $r_{p}=-0.579$ & $<0.001$ \\
\hline
\end{tabular}

Only statistically significant results are presented.

$r_{p}$, Pearson linear; $r_{s}$, Spearman rank; SBP, systolic blood pressure; BMI, body mass index; BF\%, body fat percentage; SST, subscapular skinfold thickness; W/Hip ratio, waist to hip ratio; $\mathrm{BH}$, body height; $\mathrm{BW}$, body weight; WC, waist circumference; $\mathrm{HC}$, hip circumference; MUAC, middle upper arm circumference; dHGS, dominant handgrip strength; nHGS, nondominant handgrip strength; BMR, basal metabolic rate; LBM\%, lean body mass percentage; BW\%, body water percentage; DBP, diastolic blood pressure. 
Table 3. Numerical results from analyzing the correlation between the anthropometric parameters and differences in HR and DBP between the tilt and supine body positions

\begin{tabular}{lcr}
\hline Variable & Correlation coefficient $\left(r_{p}\right)$ & $P$ \\
\hline$\Delta$ HR & & \\
BMI & 0.549 & 0.001 \\
W/Hip ratio & 0.456 & 0.009 \\
BH & 0.589 & $<0.001$ \\
BW & 0.628 & $<0.001$ \\
WC & 0.605 & $<0.001$ \\
HC & 0.574 & $<0.001$ \\
MUAC & 0.541 & 0.001 \\
dHGS & 0.610 & $<0.001$ \\
nHGS & 0.472 & 0.007 \\
BMR & 0.599 & $<0.001$ \\
$\Delta$ DBP & & \\
BMI & 0.390 & 0.027 \\
BW & 0.405 & 0.021 \\
BF\% & 0.375 & 0.035 \\
MUAC & 0.368 & 0.038 \\
dHGS & 0.437 & 0.014 \\
nHGS & 0.451 & 0.011 \\
TST & 0.366 & 0.039 \\
LBM\% & -0.383 & 0.031 \\
BW\% & -0.371 & 0.036 \\
\hline
\end{tabular}

Only statistically significant results are presented.

$H R$, heart rate; DBP, diastolic blood pressure; $r_{p}$, Pearson linear; $\Delta H R$, difference in $H R$; $\mathrm{BMI}$, body mass index; W/Hip ratio, waist to hip ratio; $\mathrm{BH}$, body height; $\mathrm{BW}$, body weight; WC, waist circumference; $\mathrm{HC}$, hip circumference; MUAC, middle upper arm circumference; dHGS, dominant handgrip strength; $\mathrm{nHGS}$, nondominant handgrip strength; BMR, basal metabolic rate; $\triangle \mathrm{DBP}$, difference in DBP between the tilt and supine body positions; BF\%, body fat percentage; TST, triceps skinfold thickness; LBM\%, lean body mass percentage; BW\%, body water percentage.

\section{Markers of obesity correlate positively with tilted SBP and DBP}

Similar to the results from the supine phase, markers of obesity (BMI, BF\%, SST, W/Hip ratio) were significantly and positively correlated with SBP during the tilt-up phase. Other anthropometric measures (BH, BW, WC, HC, MUAC), functional measures (dHGS, nHGS), and BMR were also positively correlated with SBP during the tilt-up phase. Respondents with lower LBM\% and BW\% had lower SBP during the tilt-up phase.

Unlike in the supine phase, DBP was significantly and positively correlated with markers of obesity (BMI, BF\%, SST, W/Hip ratio) in the tilt-up phase. Furthermore, other anthropometric measures (BH, BW, WC, HC, MUAC), functional measures (dHGS, nHGS), and $\mathrm{BMR}$ were also positively correlated with $\mathrm{DBP}$ in the tilt-up
Table 4. Difference in results of the ANS testing considering BMI

\begin{tabular}{lrrc}
\hline Variable & Median & No. of subjects & $P$ \\
\hline$\Delta H R(\mathrm{bpm})$ & & & 0.003 \\
$\mathrm{BMl}<25 \mathrm{~kg} / \mathrm{m}^{2}$ & 10.55 & 24 & \\
$\mathrm{BMl} \geq 25 \mathrm{~kg} / \mathrm{m}^{2}$ & 21.95 & 8 & \\
$\mathrm{SBP}$ at rest $(\mathrm{mmHg})$ & & & 0.013 \\
$\mathrm{BMl}<25 \mathrm{~kg} / \mathrm{m}^{2}$ & 113.45 & 24 & \\
$\mathrm{BMl} \geq 25 \mathrm{~kg} / \mathrm{m}^{2}$ & 140.55 & 8 & \\
$\mathrm{SBP}$ in the tilt-test $(\mathrm{mmHg})$ & & & 0.006 \\
$\mathrm{BMl}<25 \mathrm{~kg} / \mathrm{m}^{2}$ & 117.00 & 24 & \\
$\mathrm{BMl} \geq 25 \mathrm{~kg} / \mathrm{m}^{2}$ & 135.25 & 8 & \\
$\mathrm{DBP}$ in the tilt-test $(\mathrm{mmHg})$ & & & 0.045 \\
$\mathrm{BMl}<25 \mathrm{~kg} / \mathrm{m}^{2}$ & 77.55 & 24 & \\
$\mathrm{BMI} \geq 25 \mathrm{~kg} / \mathrm{m}^{2}$ & 90.05 & 8 & \\
\hline
\end{tabular}

ANS, autonomic nervous system; $B M I$, body mass index; $\Delta H R$, difference in heart rate; $\mathrm{SBP}$, systolic blood pressure; DBP, diastolic blood pressure.

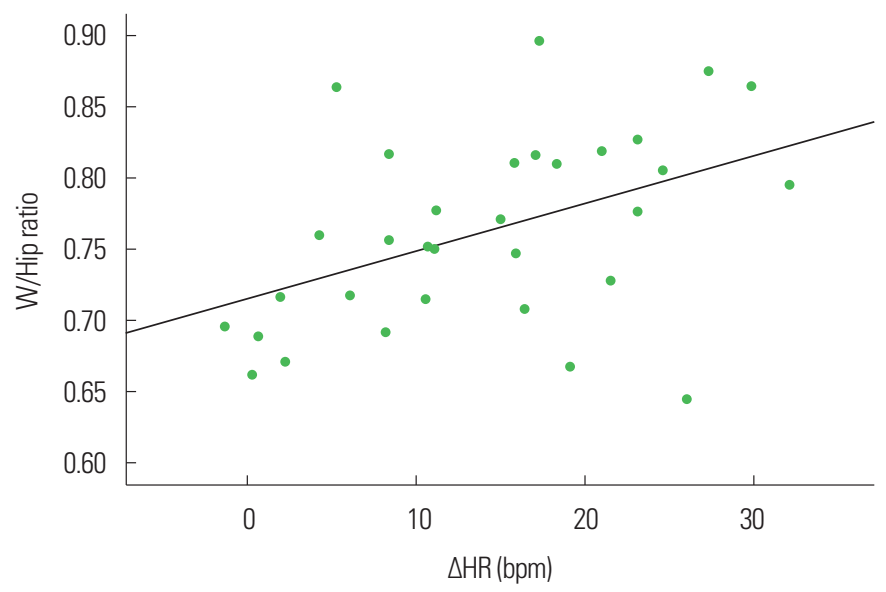

Figure 1. Correlation between the waist to hip ratio (W/Hip ratio) and the difference in heart rate $(\Delta \mathrm{HR})$ between the tilt and supine body positions.

phase. There was a negative correlation between DBP and BW\%. These numerical results are presented in Table 2.

\section{Markers of obesity correlate positively with differences in HR} and DBP between the tilted and supine phases

There was a positive correlation between difference in $\Delta \mathrm{HR}$ and markers of obesity, such as BMI (Supplementary Fig. 1), W/Hip ratio (Fig. 1), and the functional marker dHGS. A significant positive correlation with $\triangle \mathrm{HR}$ was also found for other anthropometric measures (BH, BW, WC, HC, MUAC), nHGS, and BMR. Similarly, $\triangle \mathrm{DBP}$ correlated positively with BMI, BW, BF\%, MUAC, dHGS, $\mathrm{nHGS}$, and TST. We found a negative correlation between $\triangle \mathrm{DBP}$ and $\mathrm{BF} \%\left(\mathrm{r}_{\mathrm{p}}=-0.383, P=0.031\right)$ and $\mathrm{BW} \%\left(\mathrm{r}_{\mathrm{p}}=-0.371, P=0.036\right)$. 
These numerical results are presented in Table 3. We found no significant correlations for $\triangle \mathrm{SBP}$.

\section{Lower values of tilted HR, SBP, and DBP are observed in participants with $B M I<25 \mathrm{~kg} / \mathrm{m}^{2}$}

Participants with BMI $<25 \mathrm{~kg} / \mathrm{m}^{2}$ had statistically significantly lower values of $\triangle \mathrm{HR}$ and $\mathrm{DBP}$ in the tilt-table test, $\mathrm{SBP}$ at rest, and $\mathrm{SBP}$ in the tilt-test compared with participants who had BMI $\geq 25$ $\mathrm{kg} / \mathrm{m}^{2}$ (Table 4).

\section{VR correlates positively with $\mathrm{BH}$ and $\mathrm{nHGS}$}

$$
\text { BH }\left(\mathrm{r}_{\mathrm{p}}=0.389, P=0.034\right) \text { and dHGS }\left(\mathrm{r}_{\mathrm{p}}=0.424, P=0.022\right) \text { had }
$$

a statistically significant, positive correlation with the VR. No significant correlations were observed for RSA.

\section{LF/HF, as a marker of sympathetic-vagal balance, correlates} positively with $\mathrm{HS}$

We performed separate HRV analyses for the supine phase, first 5 minutes, 2nd-7th minute, and last 5 minutes, and we compared the supine and tilt phases. Only statistically significant results are presented. In the supine position, ABSI correlated negatively with SDNN ( $\left.\mathrm{r}_{\mathrm{p}}=-0.364, P=0.044\right)$ and LF during the rest period $\left(\mathrm{r}_{\mathrm{p}}=-0.513, P=0.003\right)$. During the first 5 minutes of the tilt, the LF/HF correlated positively with dHGS $\left(r_{p}=0.370, P=0.040\right)$ and nHGS $\left(\mathrm{r}_{\mathrm{p}}=0.367, P=0.042\right)$ in the 5th minute. From the 2nd7th minute of the tilt-test, pNN50 correlated positively with SST $\left(\mathrm{r}_{\mathrm{s}}=0.350, P=0.049\right)$, and LF/HF correlated positively with dHGS $\left(\mathrm{r}_{\mathrm{p}}=0.365, P=0.044\right)$, nHGS $\left(\mathrm{r}_{\mathrm{p}}=0.375, P=0.038\right)$, and LBM\% $\left(r_{p}=0.352, P=0.048\right)$. During the last 5 minutes of the tilt-test, the LF/HF correlated positively with dHGS $\left(\mathrm{r}_{\mathrm{p}}=0.376, P=0.037\right)$ and BMR $\left(\mathrm{r}_{\mathrm{p}}=0.365, P=0.040\right)$. We found statistically significant differences between the supine and tilt test positions in the pNN50, r-MSSD, HF, and HF/LF HRV variables. These results are presented in Supplementary Table 6.

\section{BF\% is an independent positive predictor and BW\% is an}

\section{independent negative predictor of tilted SBP}

We performed univariable and multivariable regression analyses to determine which anthropometric and body composition measures best predict tilted SBP (Table 5 and Supplementary Table 7).
Table 5. Results of the multivariable logistic regression models

\begin{tabular}{lrcc}
\hline Model & \multicolumn{1}{c}{$\beta$} & $95 \%$ Cl for $\beta$ & $P$ \\
\hline Model 1 & & & \\
Age & 0.592 & -2.580 to 3.763 & 0.705 \\
Sex & -10.718 & -24.270 to 2.834 & 0.116 \\
BMl $\geq 25 \mathrm{~kg} / \mathrm{m}^{2}$ & 9.516 & -6.925 to 25.957 & 0.245 \\
BF\% & 0.993 & 0.070 to 1.916 & 0.036 \\
Model 2 & & & \\
Age & -0.720 & -3.558 to 2.118 & 0.607 \\
Sex & 16.164 & -2.570 to 34.898 & 0.088 \\
BMl $\geq 25 \mathrm{~kg} / \mathrm{m}^{2}$ & 2.078 & -14.654 to 18.811 & 0.801 \\
MUAC & 3.420 & 1.245 to 5.596 & 0.003 \\
Model 3 & & & \\
Age & 0.588 & -2.578 to 3.753 & 0.706 \\
Sex & -10.669 & -24.186 to 2.847 & 0.117 \\
BMl $\geq 25 \mathrm{~kg} / \mathrm{m}^{2}$ & 9.403 & -7.047 to 25.852 & 0.251 \\
BW\% & -1.370 & -2.634 to 0.106 & 0.035 \\
\hline
\end{tabular}

$\mathrm{Cl}$, confidence interval; $\mathrm{BMl}$, body mass index; $\mathrm{BF} \%$, body fat percentage; MUAC, middle upper arm circumference; BW\%, body water percentage.

Because of our small number of participants, we calculated several regression models with a maximum of four variables each. In the first and second models, respectively, BF\% and MUAC were positive predictors of tilted SBP independent of age, sex, and BMI $\geq 25$ $\mathrm{kg} / \mathrm{m}^{2}$. In the third model, BW\% was a negative predictor of tilted SBP independent of age, sex, and BMI $\geq 25 \mathrm{~kg} / \mathrm{m}^{2}$.

\section{DISCUSSION}

The results of this study show statistically significant correlations between autonomic cardiovascular regulation, as measured by changes in SBP, DBP, and HR during the tilt-table test, HR response to the Valsalva maneuver, and HRV, and anthropometric and body composition measures. During the first 10 minutes in the supine position during the tilt-test, SBP correlated significantly and positively with markers of obesity (BMI, BF\%, SST, W/H ratio, $\mathrm{W} /$ Hip ratio). Contrarily, we found a statistically significant negative correlation between SBP and LBM\% and BW\%, which are indicators of a high percentage of muscle mass, as well as between DBP and BW\%. Therefore, we speculate that in the supine rest position, fat tissue affects SBP. There are several possible explanations for our observed association between $\mathrm{BP}$ and body fat composition. One is the direct influence of fat tissue endocrine regulation on the SNS or blood vessels. ${ }^{2}$ On the other hand, higher sympathetic ac- 
tivity could cause an increase in SBP and fat accumulation. ${ }^{23}$ Our observational study cannot determine the causality of the association. Moreover, exogenous factors such as low levels of physical activity also affect SBP and fat accumulation and could explain this observation. ${ }^{24}$

We observed several correlations between the tilt-test parameters and body composition. The tilt-test is a standardized method for evaluating the ANS. By passively producing a standing position, it causes an accumulation of blood in the veins of the legs, which reduces the effective volume of circulating blood. That decreases venous inflow and stroke volume and consequently lowers BP, which activates the SNS by means of the baroreceptor reflex. Higher SNS activity increases vasoconstriction of the arteries and vascular resistance, leading to an increase in BP. In this study, we found statistically significant correlations between SBP and the same anthropometric parameters during both the supine position and the passive tilt. Moreover, in a multivariable regression model, both BF\% and BW\% were independent predictors of tilted SBP. During the tilt-up phase, DBP was also statistically significantly correlated positively and negatively with the same parameters as SBP. In contrast to SBP and pulse pressure, which depend on stroke volume and aortic compliance, DBP physiologically depends predominantly on total vascular resistance. That might explain the greater increase in DBP than SBP that we observed during the passive tilt-up phase. ${ }^{25}$ Notably, during tilting, SNS increases DBP by means of vasoconstriction of the arteries, whereas SBP depends on the effective volume of circulating blood being corrected by vasoconstriction of veins and stimulation of the heart muscle. ${ }^{26}$ Higher $\triangle \mathrm{DBP}$ than $\triangle \mathrm{SBP}$ thus confirms the described theory. Because $\triangle \mathrm{DBP}$ and $\triangle \mathrm{HR}$ during tilting were significantly and positively correlated with several markers of obesity, we found no significant correlation between obesity markers and $\triangle \mathrm{SBP}$.

Another measure frequently used in evaluations of the ANS is the Valsalva maneuver, which is divided into phases that consider the predominance of the SNS and PNS. The VR, indicating cardiovagal activation, is the ratio between the highest pulse during phase II and the lowest pulse during phase IV. ${ }^{27}$ The VR correlated significantly and positively with height and dHGS. That could be an indicator of larger chest volume and muscle mass. Mechanical pressure stimulation of skeletal muscles can induce reflex changes in HR and BP, although the neural mechanisms underlying this effect are unclear. ${ }^{28}$ A recently published study showed that cardiac sympathetic nerve activity regulates $\mathrm{HR}$ responses to muscle mechanical pressure stimulation and that the direction of $\mathrm{HR}$ responses depends on the tonic level of the nerve activity, i.e., bradycardia occurs when the tonic activity is high, and tachycardia occurs when the activity is low. ${ }^{28}$ On the other hand, we found no correlation between the VR and anthropometric measurements, which are more specific markers of obesity.

The third part of this study evaluated HRV, which is considered to be a positive predictor in the prevention of cardiovascular diseases. It is highest in children and adults with good cardiorespiratory fitness status. ${ }^{29}$ We found a correlation between HRV and anthropometric measurements during both the rest and tilt phases of the tilt-test. From the HRV, we calculated SDNN and r-MSSD as markers of HRV in long cycles, pNN50 and HF as markers of the PNS, and the LF/HF which is partly a marker of the PNS but dominantly a marker of the SNS. ${ }^{21}$ The negative correlation between ABSI and the HRV markers SDNN and LF during rest indicates that PNS activity might be connected with a lower percentage of adipose tissue. ABSI is affected by BMI and height, but it mostly reflects WC, so it is better marker of obesity than BMI. The importance of these correlations is emphasized by a recent study showing that twelve weeks of aerobic exercise in women with obesity was associated with a significant improvement in HRV parameters, indicating an improvement in ANS function. ${ }^{30}$ However, connecting the decrease in PNS activation with obesity must be done with care because other measurements of cardiovagal tonus (e.g., r-MSSD, HF, VR, RSA) were not statistically significantly correlated with markers of obesity in our study.

Finally, we wanted to see whether HR, SBP, and DBP differ depending on $\mathrm{BMI} .^{31}$ In our study, the tilt-test induced almost double the increase in $\mathrm{HR}(\triangle \mathrm{HR})$ in the group of subjects whose BMI was $\geq 25 \mathrm{~kg} / \mathrm{m}^{2}$ than it did in the group whose BMI was $<25 \mathrm{~kg} / \mathrm{m}^{2}$. The group with a BMI $\geq 25 \mathrm{~kg} / \mathrm{m}^{2}$ also had significantly higher SBP during rest and higher SBP and DBP during the tilt-up phase. That is evidence of a relationship between being overweight and having a higher sympathetic/parasympathetic activity ratio, which has been already described in the literature. ${ }^{3}$ Although BMI is simple to calculate, and therefore often used, it does not provide infor- 
mation about body shape and composition. There is evidence that people with a higher $\mathrm{W} / \mathrm{Hip}$ ratio have increased insulin, glucose, adrenocorticotropic hormone, and triglyceride values and decreased growth hormone secretion peaks compared with people with a similar BMI but lower W/Hip ratio. A higher W/Hip ratio is also connected with elevated pulse pressure and HR. ${ }^{32}$ We showed here that $\Delta \mathrm{HR}$ during tilting also correlated positively with the $\mathrm{W} / \mathrm{Hip}$ ratio, which again indicates that focusing on changes in HR and DBP during tilting is important. Several previously published studies observed similar connections between obesity markers and ANS parameters. Hillebrand et al. ${ }^{16}$ observed that body fat, especially abdominal body fat, is associated with mean HR, corrected QT time, and the ventricular gradient magnitude. Furthermore, in the Olivetti Heart Study performed with 1,079 men, the multiple regression analysis showed that WC remained the strongest independent predictor of BP after adjustment for confounders. ${ }^{33}$ In results similar to ours, a study performed with medical students from different populations found that the $\mathrm{WC}$ and $\mathrm{W} /$ Hip ratio correlated with BP values. ${ }^{34,35}$ Taken together, these results indicate that abdominal obesity correlates with higher sympathetic activity and hormonal and metabolic dysregulation through the hypothalamicpituitary-adrenal axis.

This study has several limitations. It is a descriptive, cross-sectional study with a relatively small number of participants. Because of the low number of subjects, it was difficult to address the issue of plausible confounders. Nevertheless, we used a complete, standardized battery of ANS tests and thereby expanded what is known about the ways in which body composition influences ANS.

In conclusion, we found a relationship between higher sympathetic activity, as evaluated by monitoring cardiovascular regulation, and a higher amount of adipose tissue in young healthy persons. The most prominent correlation is a positive one between SBP and waist and HC. In addition, SNS activation, as mediated by the baroreflex during the tilt-up phase of the tilt-test, is also positively correlated with markers of obesity. Therefore, we suggest that in addition to measuring HR and SBP during the tilt-up stress test, clinicians and researchers should also focus on changes in DBP, which could provide additional indications of increased SNS activity in otherwise healthy persons. Remarkably, all the changes in autonomic cardiovascular regulation reported here were already found in young and healthy people, so those parameters could be used as the earliest appearing predictive markers for cardiovascular disease.

\section{CONFLICTS OF INTEREST}

The authors declare no conflict of interest.

\section{AUTHOR CONTRIBUTIONS}

Study concept and design: TS, IS, MH; acquisition of data: all authors; analysis and interpretation of data: all authors; drafting of the manuscript: TS, IS; critical revision of the manuscript: all authors; statistical analysis: MKS; administrative, technical, or material support: all authors; and study supervision: $\mathrm{MKS}, \mathrm{MH}$.

\section{SUPPLEMENTARY MATERIALS}

Supplementary Tables 1-7 and Figure 1 can be found via https:// doi.org/10.7570/jomes20041.

\section{REFERENCES}

1. Messina G, Valenzano A, Moscatelli F, Salerno M, Lonigro A, Esposito T, et al. Role of autonomic nervous system and orexinergic system on adipose tissue. Front Physiol 2017;8:137.

2. Bray GA. Obesity, a disorder of nutrient partitioning: the MONA LISA hypothesis. J Nutr 1991;121:1146-62.

3. Guarino D, Nannipieri M, Iervasi G, Taddei S, Bruno RM. The role of the autonomic nervous system in the pathophysiology of obesity. Front Physiol 2017;8:665.

4. Garg R, Malhotra V, Goel N, Dhar U, Tripathi Y. A study of autonomic function tests in obese people. Int J Med Res Health Sci 2013;2:750-5.

5. Costa J, Moreira A, Moreira P, Delgado L, Silva D. Effects of weight changes in the autonomic nervous system: a systematic review and meta-analysis. Clin Nutr 2019;38:110-26.

6. Fidan-Yaylali G, Yaylali YT, Erdogan Ç, Can B, Senol H, GedikTopçu B, et al. The association between central adiposity and autonomic dysfunction in obesity. Med Princ Pract 2016;25: 
442-8.

7. Lewis JE, Tannenbaum SL, Gao J, Melillo AB, Long EG, Alonso Y, et al. Comparing the accuracy of ES-BC, EIS-GS, and ES Oxi on body composition, autonomic nervous system activity, and cardiac output to standardized assessments. Med Devices (Auckl) 2011;4:169-77.

8. Guiraud T, Labrunee M, Gaucher-Cazalis K, Despas F, Meyer P, Bosquet L, et al. High-intensity interval exercise improves vagal tone and decreases arrhythmias in chronic heart failure. Med Sci Sports Exerc 2013;45:1861-7.

9. Naci H, Salcher-Konrad M, Dias S, Blum MR, Sahoo SA, Nunan D, et al. How does exercise treatment compare with antihypertensive medications? A network meta-analysis of 391 randomised controlled trials assessing exercise and medication effects on systolic blood pressure. Br J Sports Med 2019;53: 859-69.

10. Koenig J, Windham BG, Ferrucci L, Sonntag D, Fischer JE, Thayer JF, et al. Association strength of three adiposity measures with autonomic nervous system function in apparently healthy employees. J Nutr Health Aging 2015;19:879-82.

11. Vanderlei LC, Pastre CM, Freitas Júnior IF, Godoy MF. Analysis of cardiac autonomic modulation in obese and eutrophic children. Clinics (Sao Paulo) 2010;65:789-92.

12. Lourenço Dias AR, de Souza KA, de Jesus Lima de Sousa LC, Dos Santos KM, Kolesny Tricot G, de Araújo JA, et al. Higher blood pressure and lower cardiac vagal activity in obese young individuals in supine and seated position. J Clin Transl Res 2017;3:328-37.

13. Rossi RC, Vanderlei LC, Gonçalves AC, Vanderlei FM, Bernardo AF, Yamada KM, et al. Impact of obesity on autonomic modulation, heart rate and blood pressure in obese young people. Auton Neurosci 2015;193:138-41.

14. Barreto GS, Vanderlei FM, Vanderlei LC, Leite ÁJ. Impact of malnutrition on cardiac autonomic modulation in children. J Pediatr (Rio J) 2016;92:638-44.

15. Izumi M, Manabe E, Uematsu S, Watanabe A, Moritani T. Changes in autonomic nervous system activity, body weight, and percentage fat mass in the first year postpartum and factors regulating the return to pre-pregnancy weight. J Physiol Anthropol 2016;35:26.
16. Hillebrand S, de Mutsert R, Christen T, Maan AC, Jukema JW, Lamb HJ, et al. Body fat, especially visceral fat, is associated with electrocardiographic measures of sympathetic activation. Obesity (Silver Spring) 2014;22:1553-9.

17. Molfino A, Fiorentini A, Tubani L, Martuscelli M, Rossi Fanelli F, Laviano A. Body mass index is related to autonomic nervous system activity as measured by heart rate variability. Eur J Clin Nutr 2009;63:1263-5.

18. Drulović J, Gavrilović A, Crnošija L, Kisić-Tepavčević D, Krbot Skorić M, Ivanović J, et al. Validation and cross-cultural adaptation of the COMPASS-31 in Croatian and Serbian patients with multiple sclerosis. Croat Med J 2017;58:342-8.

19. Novak P. Quantitative autonomic testing. J Vis Exp 2011;(53): 2502.

20. Berntson GG, Cacioppo JT, Quigley KS. Respiratory sinus arrhythmia: autonomic origins, physiological mechanisms, and psychophysiological implications. Psychophysiology 1993;30: 183-96.

21. Shaffer F, Ginsberg JP. An overview of heart rate variability metrics and norms. Front Public Health 2017;5:258.

22. World Health Organization. Obesity: preventing and managing the global epidemic. Report of a WHO consultation (WHO technical report series 894). Geneva: World Health Organization; 2000.

23. Thorp AA, Schlaich MP. Relevance of sympathetic nervous system activation in obesity and metabolic syndrome. J Diabetes Res 2015;2015:341583.

24. Fasting MH, Nilsen TI, Holmen TL, Vik T. Life style related to blood pressure and body weight in adolescence: cross sectional data from the Young-HUNT study, Norway. BMC Public Health 2008;8:111.

25. Green DJ, Hopman MT, Padilla J, Laughlin MH, Thijssen DH. Vascular adaptation to exercise in humans: role of hemodynamic stimuli. Physiol Rev 2017;97:495-528.

26. Sheng Y, Zhu L. The crosstalk between autonomic nervous system and blood vessels. Int J Physiol Pathophysiol Pharmacol 2018;10:17-28.

27. Pstras L, Thomaseth K, Waniewski J, Balzani I, Bellavere F. The Valsalva manoeuvre: physiology and clinical examples. Acta Physiol (Oxf) 2016;217:103-19. 
28. Watanabe N, Hotta $\mathrm{H}$. Heart rate changes in response to mechanical pressure stimulation of skeletal muscles are mediated by cardiac sympathetic nerve activity. Front Neurosci 2017;10: 614.

29. Bellenger CR, Fuller JT, Thomson RL, Davison K, Robertson EY, Buckley JD. Monitoring athletic training status through autonomic heart rate regulation: a systematic review and metaanalysis. Sports Med 2016;46:1461-86.

30. Park HY, Jung WS, Kim J, Hwang H, Lim K. Twelve weeks of aerobic exercise at the lactate threshold improves autonomic nervous system function, body composition, and aerobic performance in women with obesity. J Obes Metab Syndr 2020; 29:67-75.

31. Taghizadeh N, Boezen HM, Schouten JP, Schröder CP, Elisabeth de Vries EG, Vonk JM. BMI and lifetime changes in BMI and cancer mortality risk. PLoS One 2015;10:e0125261.
32. Ljung T, Holm G, Friberg P, Andersson B, Bengtsson BA, Svensson J, et al. The activity of the hypothalamic-pituitary-adrenal axis and the sympathetic nervous system in relation to waist/ hip circumference ratio in men. Obes Res 2000;8:487-95.

33. Siani A, Cappuccio FP, Barba G, Trevisan M, Farinaro E, Lacone $\mathrm{R}$, et al. The relationship of waist circumference to blood pressure: the Olivetti Heart Study. Am J Hypertens 2002;15: 780-6.

34. Chaudhary S, Alam M, Singh S, Deuja S, Karmacharya P, Mondal M. Correlation of blood pressure with body mass index, waist circumference and waist by hip ratio. J Nepal Health Res Counc 2019;16:410-3.

35. Nkeh-Chungag BN, Mxhosa TH, Mgoduka PN. Association of waist and hip circumferences with the presence of hypertension and pre-hypertension in young South African adults. Afr Health Sci 2015;15:908-16. 